straight in little more than a fortnight; and the child left the hospital at the end of March, with orders to wear some straight leather side-splints and a bandage for a long while.]

7. The ultimate consequences of disease of the knee-joint have been shown you in John $\mathrm{H}$ limb I have been obliged to amputate, and who is going to leave the hospital this week. His disease was probably inflammation of the synovial membrane, followed by ulceration of the cartilages and necrosis of the tibia, in consequence of a strumous constitution, but it may have been originally scrofulous disease of the tibia spreading secondarily to the joint.

This man told us that his knee began to swell six months ago, but was cured by blisters, till three months ago, when it again swelled, but was a second time made much better, till three days before his admission, when swelling returned. He has, throughout most of this time, had startings at night and a good deal of pain, and very early in the case an abscess formed below the outer part of the joint, which has never healed. On his admission on the 11th of December last, there was a small opening below the head of the tibia, on the outside, into which the probe passed to some depth, from which there was a watery discharge; there was a good deal of thickening all round the jnint, and some fluid within it, which I thought was an abscess, prominent chiefly on the inside of the femur, but communicating probably with the external opening by a narrow sinus; and the man was so weak and emaciated that I told you I could not expect to cure the disease.

You may remember, however, how much relief was at first obtained by rest, by good living, and by saline and opium, followed by calumba and ammonia, and then by quinine; he lost the pain and starting in great measure, and the fluid lessened considerably. In three weeks' time, howerer, a bad cough came on, with perspirations, and some return of pain in the joint, with evidence of suppurative inflammation within it, afforded by enlargements of the superficial veins of the inner part of the thigh.

At this time, Dec. 30th, I put him under the physician's care for his cough, whose opinion was against the probable existence of tubercles, and on January 23rd, I amputated the limb, at which time the original swelling on the inside had re-appeared, and a large separate abscess formed on the outside of the thigh towards the ham. You may remember, that on examination, matter was found in the joint: besides a large foul abscess in the thigh, communicating with it by a small opening, it was found that the original sinus led to a portion of dead bone, the upper end of which was seen in the head of the tibia within the joint, the cartilages of all these bones being in great measure absorbed by nlceration. It is not my intention to notice anything further in this man's case, who is now well; but it is a good example of the effects which may follow disease of this joint, let its origin have been whatever it may; and only amputation can preserve life in such a stage as this, when every texture of the joint is at once affected.

8. In such cases as the two next, it is sometimes difficult to decide in which texture the disease originated, but probably in great measure in the ligamentous tissue around the joint; and such cases as these are sometimes very tedious, and not easily cured, from their tendency to relapse as soon as the patients are allowed to get up and use the joints.

The first of these is Frances $\mathrm{H}$ mitted on the 8th of January; she is a honsemaid, and is obliged to kneel much, but there is no evidence of her having had the usual bursal swelling from this cause She has suffered from pain and swelling of the knee during the last nine months, and has har leeches and blisters applied before she came into the hospital, at which time there was slight general fulness of the parts around the joint, which was tender and painful, but there was no synovial swelling.

Being a nervous person, I first wrapped the joint up in belladonva plaster on leather, with a bandage over it, and gave her steel, and aloes and myrrh pill; but finding that there was distinctly the pain of disease, and not hysterical pain only, I applied a blister on the $20 \mathrm{th}$, and have re peated it three times since that time, nnder the use of which the joint has much improved, but a little pain and swelling still remain.

After this, the joint was enclosed in leather straps, with mercurial plaster and a bandage, and the patient left, apparently cured, at the end of March.

9. You may see the same affection, in a rather more aggra vated form, in the case of Thomas $D$, thirty-seven years of age, admitted on the 8 th of January, the same day as the last patient; and in him, also, the disease began about nine months previously, and had, as in her, been prevented from getting well by his continuing to follow his employment. In this circumstance, we see one of the greatest disadvantages which those of all classes labour under who depend on their daily exertions; and it is not wonderful that, when neglected, such cases as these two may sometimes be seen to terminate, notwithstanding their present chronic condition, in ulceration of the cartilages and a succession of small abscesses, and, finally, in the loss of the limb.

It appeared, from his account, that nine months ago pain came on in his knee without any apparent cause, followed in about a fortnight by some swelling; that a blister on each side of the joint relieved the pain, but the swelling continued; that two months ago he came here as an out-patient, and that some more blisters had reduced the swelling and lessened the pain; but that all this time he had continued to go about as a carman.

On his admission there was a good deal of pain, increased by using the joint, and there was puffy swelling and hardness round the joint, with considerable stiffness; but there was no synovial enlargement, and none of the starting of diseased cartilages. He has therefore had a succession of several blisters to his knee, and our notes tell us that the knee is in all respects better. I do not consider him, however, by any means cured; and he will probably require rest, either in bed or by means of splints, for a long while, and when allowed to use the joint again he must exercise the greatest caution.

In such cases as these you require several measures to get rid of the thickened condition of the soft parts, on which the stiffness depends; of which, iodine lotion, or hydrochlorate-of ammonia lotion, or local vapour baths, or shampooing, or effusion of water from a height, as from a pump or douche, are the best.

[This man continued to get better till he went out, on April 9th, and as the swelling subsided it became evident that there was thickening of some parts of the synovial membrane, feeling like semi-cartilaginous masses moveable with the membrane to which they were attached.]

10. The last case I invite you to observe, is that of a girl named $\mathrm{J} \longrightarrow$, but $I$ have only time to allude to it as illustrating a not unfrequent joint affection, in which, with the most severe pain, so that she cannot bear to be touched or moved, there is yet no real disease; it is hysterical pain only of her hip and knee of a very aggravated kind, depending on her general health. At the same time, never forget that acute hysterical pain is often added to real disease, so as to mask and conceal its symptoms; and let your proceedings be cautiously directed, lest you increase the primary affection by that exercise of the joint which the hysteria would require, if alone.

\section{PERIOSTEAL DISEASE AFFECTING THE DURA MATER.}

By R. H. GOOLDEN, M.D. Oxon., F.R.C.P. ASSISTANT-PKXSICIAN TO ST, THOMAS'S HOSPITAL

1. Frequency of its occurrence. 2. Symptoms. 3. Autopsy of a patient suffering from this affection, killed by an accident. 4. Its identity with nodal periostitis. 5. The severity of its form as it occurs amongst sailors and tropical residents. 6. Not confined to them. 7. Diagnosis. 8. Treatment; resuits. 9. Occasional connexion with epilepsy and paralysis. 10. Cases illustrative of such connexion. 11. Demonstration of the identity of cause in the cases quoted. 12. These cases were not syphilitio in their origin. 13. The assertion is made from induction from a large number of facts under peculiar advantages for observation.

1. ThE dura mater is supposed to be very little liable to disease, except as the result of injury, or in connexion with strumous softening of the petrous portion of the temporal bone, or with abscesses about the ear, or as the effect of the syphilitio poison; and this notion, supported by some of our best authorities, seems to have diverted attention from a very common affection of this membrane, of the same character as the periostitis which gives rise to nodes on the tibia or cranial bones

This partial affection of the dura mater, to which I wish to call the attention of the profession, may or may not coexist with general periosteal disease. It is part of the same morbid state, and it has no necessary connexion either with syphilis or with mercury, though these poisons, and especially the latter, greatly favour its development. Indeed the worst cases that I have an opportunity of observing, have been those who, in the tropics, have taken large quantities of mercury for 
fevers or hepatic disease, often many years previously, and have been afterwards exposed to cold and damp in our more northern latitudes.

2. When I held office at the hospital-ship Dreadnought, these cases were both very severe and very common, and I have no doubt continue to be so. They give way, at least for the time, to very simple treatment-viz., iodide of potassa, with sarsaparilla, blisters or issues to the head, and full doses of opium every night, with a good nutritious diet-in the same way as periosteal nodes are known to do. When the pericranium is the seat of the disease, it is at once recognised by the presence of great tenderness, as well as unevenness to the touch; but when the dura mater is affected, this morbid condition is not so palpable; it is, however, evinced by unmistakable symptoms. The patients are often free from pain during the day, but at eight or nine o'clock at night the pain sets in, and is so agonizing that they are willing to submit to any measure for relief. It lasts till three or four o'clock in the morning, followed by a profuse perspiration, when the patient gets some sleep. The intermittent character, and the time and duration of the paroxysms, are very remarkable. For some time the cerebral functions are not impaired, but at length the sufferer complains of a constant headache, though not so severe as during the paroxysm; he holds his head forward, with his brows knit; then follow occasionally epileptic fits, weakness of the lower limbs, paralysis, and the patient becomes greatly emaciated, despondent, and suffers colliquative perspirations.

3. Some years ago, a sailor, who had frequently been a patient in the Dreadnought for such attacks as I have described, was accidentally killed by falling between two ships in the river. This afforded Mr. Busk, my colleague, an opportunity of examining the head, when it was found that the inner table of the skull was greatly thickened, and also the dura mater.

4. Such is the most marked and aggravated form of the disease occurring in patients debilitated by climate, mercury, purpura, or syphilis, which no one would fail to recognise as identical (except in situation) with periosteal disease, when it attacks the tibia, ulna, cranial bones, crest of the ilium, \&c., in the form of nodes, and for which the iodide of potassium seems to be the appropriate if not specific remedy.

5. Although this aggravated form of periosteal disease of the dura mater is most common in sea-port hospitals, where the patients have been for the most part exposed to all the predisposing causes, yet observation has convinced me that it occurs also in a milder form, and therefore more likely to escape recognition, as the cause of many distressing headaches, ultimately leading to epilepsy or paralysis, or at all events reducing the sufferer to the most distressing state of debility; and patients so suffering apply to us for relief in our daily practice, both in hospitals and in private life. Such cases I have met with, both as out and in-patients at St. Thomas's Hospital, as servants in the employment of the Great Western Railway, and in private practice.

6. These cases are marked by headache, having its regular nightly paroxysms, morning perspirations, and gradual emaciation. The patients have mostly been treated for dyspepsia, rheumatism, gout, neuralgia, brow ague, and often for phthisis. I have several times been called upon to examine such patients' chests, as they were supposed to be obscure cases of phthisis, having, in addition to the above symptoms, some cough and bronchial murmurs; but by the peculiar expression of countenance, the pain and wakefulness during the first part of the night, the true nature of the disorder has been recognised.

7. A blister to the head, opium at night, and iodide of potassium and sarsaparilla during the day, have dissipated all the symptoms, and the patients have returned to their usual occupations.

8. It is interesting to know what becomes of these patients. The great drawback to the results of our hospital practice is, that we cannot infer that a patient is permanently cured because we do not see him again. On the Great Western Railway I have had an opportunity of watching the results. I have found that some have had no recurrence of the attack; others are subject to relapses after various intervals, but are always relieved by the same remedies; others are attacked by periostitis in its more generally recognised form; others are relieved only so long as they continue to take the iodide of potassium.

9. The connexion of this disease with epilepsy is often more intimate than is generally supposed. Without pretending that it is an universal cause, as we know that intestinal irritation and direct injury to the bones will produce epilepsy, it is as well, in obscure cases, to bear in mind that this is a possible cause, and one capable of being to a considerable extent, if not entirely, removed.

10. I have at present two cases of epilepsy under treatment in St. Thomas's Hospital, which I refer to this cause. The first, a man, in Luke's ward, No. 3, admitted February 25th, 1851 ; seaman. Epileptic fits occurring at various intervals, the last on Saturday; constant headache; no sleep; profusely perspiring, and becoming very much emaciated. Has been ill eight months; was in Guy's Hospital for three months, and left it a month ago. He was then quite paralyzed on the left side; he had a moxa on the crown of the head, and electricity applied, and he nearly recovered the use of his limbs; but since he left the hospital he has become much worse, and is suffering more frequently from fits. Tongue clean, white, and indented with the teeth; pulse feeble; sight pretty good, pupils dilated; bowels constipated. Diagnosis: epilepsy with paralysis. Turpentine and castor oil, of each half a drachm, to be taken directly.

Feb. 27th.-No worms passed, but a large quantity of pale and lumpy fæces; he was very sick, and felt very cold all night. Calomel, two grains; rhubarb, five grains, every night; decoction of pomegranate, one ounce and a half; liquor potassæ, half a drachm, thrice daily.

March 1st.-Complains greatly of the head, especially at night; has had a fit every day since the last visit; bowels open, and fæces bright-yellow. Sixteen ounces of blood to be taken from the nape of the neck by cupping.

6th.- No change of symptom; felt a little relief after the cupping, but it was not permanent. It was thought better to change the plan of treatment. Meat diet. Zinci valerianatis, two grains, three times a day in a pill.

26 th. - The headache is worse, especially at night; head hot; no appetite; sweating profusely, and the fits occur twice a day; the paralysis increasing, and he is becoming imbecile; bowels rather constipated. Calomel, two grains; compound jalap powder, half a drachm, on alternate mornings; spirit lotion to the head; hair to be cut close.

29th.- No improvement whatever, always worse in the first part of the night. Under the idea that this case might be connected with disease of the dura mater, I prescribed as follows:-Soap and opium pill, five grains, every night; iodide of potassa, five grains; liquor potassa, half a drachm; compound decoction of sarsaparilla, one ounce and a half, thrice a day.

April 3rd.-Nothing can be more marked than the inprovement; he has had no fit since the last visit; sleeps well; perspirations much less; he has still constant pain in the head, especially over the left brow, but the paroxysm is hardly perceptible at night; pain in the back and loins; bowels open. Shave the head and apply a blister. Porter, one pint daily.

16th.-The improvement has been constant and gradual. The blister was repeated to the scalp, and he has now a very slight pain over the left brow; pain in the loins; he has recovered his intelligence; looks and speaks cheerfully, and considers himself better than he has been for the last eight months. He cannot support himiself without crutches, as his lower limbs are not only weak but somewhat paralyzed, but he is daily improving in that respect.

June.-I have been unwilling to discharge the patient until now, as he has been improving in health and strength. $\mathrm{He}$ has continued the medicine up to this time, and now has no complaint. No epileptic fit has occurred since the 29 th of March; as he walks there is a slight dragging of the left leg. He will go out on Tuesday.

The next case that I shall refer to is that of a girl, Deliah Williams, aged eighteen; single; admitted in Mary's ward. She is chlorotic, and has only once menstruated, suffering severe headaches in nightly paroxysms, and epileptic fits every two or three days; profuse perspirations. There is dulness of intellect, which her friends say was not formerly the case. She was ordered, iodide of potassium, five grains; liquor potassa, half a drachm; compound decoction of sarsaparilla, one ounce and a half, three times a day. The head was shaved and blistered several times, and the result has been that the headaches have left hor, and she has only had two fits since she has been taking the medicine.

$I$ shall refer to a case of the same character, illustrating the connexion between cerebral symptoms and periosteal disease. A guard on the Great Western Railway had been under my care for general periosteal disease, some years ago. He was a very tall, thin man; had been in the army, and ho was dis. abled for a time from the railroad service by the loss of the use of the right arm, from an affection of the shoulder-joint, which was relieved by the iodide of potassium, and a nitric- 
acid issue; he had likewise nodes on the clavicle and sternum, with night pains and perspirations. This man returned to his occupation as guard, and remained well for six months, after which he was suddenly seized with what was supposed to be an attack of apoplexy. He was insensible at the time, and was found early in the morning fallen from the bed, with loss of sight and strabismus; the mouth drawn on one side. He soon recovered under the use of iodide of potassium and sarsaparilla, and the application of blisters to the head. The previous history was the guide in this interesting case, which occurred under the care of Mr. Champneys, of Slough, with whom I saw it.

There was another instance of cerebral irritation lately under my care, in Luke's ward, St. Thomas's Hospital, presenting some points of interest.

John B- No. 4, Luke's ward, aged seventeen, had been engaged in black-lead works; admitted Feb. 18, 1851, an engine driver; had been ill for three years, and imagined that he had got the black-lead into his bowels. Tenesmus, passing chiefly blood and mucus as often as sixteen times a day; bowel comes down; tongue natural; pulse soft and regular; skin moist; headache; no appetite. He was treated for dysentery: calomel and castor-oil; leeches to the anus; opium and aromatic mixture, with ipecacuanha. The purging continued till the 25 th, (i.e., one week, and all that now passes from the bowels seems to be pure bile.

27th.-He had a motion to-day, attended with great pain, and he described what passed as being like a mass of black pills, and very hard. The abdomen was soft, not sunken, and there is nothing to indicate mischief. He was ordered calomel and castor-oil, which brought a way a large quantity of claycoloured faces in a large mass, and pretty firm, followed by a proper, loose, bilious matter.

April 5th.- Without any apparent cause he was seized with a most severe rigor and pain in the abdomen, and general convulsions. The first impression was, that the contents of the bowels had escaped into the peritonæum, and that the rigor was the consequence; the slightest movement increased the pain, and we could not get his attention to answer questions; his countenance showed an expression of extreme terror. There was no heat of skin or quickness of pulse beyond what would be accounted for by the convulsive efforts, and no sign of collapse. He was treated with opium in large and frequent doses; leeches and fomentations to the abdomen; calomel and castor-oil. In the morning of the following day he had a remission of spasm, and got some sleep, which we attributed to the opium; the bowels were opened, and what passed was semi-solid and clayey. The convulsion returned as severely as ever, and he took musk, two grains; camphor, one grain; watery extract of aloes, one grain, every four hours.

8th.-Convulsion fits continue at short intervals; complains of great pain in the abdomen, which is hard and knotty during the spasm, and quite soft during the remissions; stools quite black and copious; lumpy; pulse neither excited nor sppressed; great pain in the head. Calomel, five grains, opium, one grain directly; castor-oil in the evening; cut the hair; musk, two grains; camphor, one grain, three times in the day.

17 th.-As the symptoms have continued unchanged, I do not mention the detail beyond observing that the stools became natural and bilious under such treatment as was calculated to allay any irritation, and yet promote the proper secretion of the chylopoietic viscera. He has had no appetite for food, and from the headache he complained of, $I$ had discontinued any opiate for a time, but still the spasms and convulsions continue, and he takes the musk and camphor. The fear was that the boy would sink from pain. I ordered a blister to the head.

19th.-On my visit to-day I find $\mathrm{B}$ had no more couvulsion since the middle of the night, when the blister began to draw. Has slept well, and calls for the first time for food. Has no pain in head or abdomen; bowels have been relieved, but he is very feeble.

From this time he gradually regains his strength and takes meat and wine, and continues the musk and camphor, with occasional doses of castor-oil; but there is always an anxious expression of countenance, and he does not sleep well during the first part of the night, and suffers some headache.

May 12th-Complains of pain in the abdomen, which comes on in paroxysms, and whilst the pain is present the abdomen is hard and knotty; anxiety of expression increased; meat and wine omitted; resume the musk and camphor.

17th.-He has been attacked in the night with the spasms as severely as before; has had no sleep, and he has taken two half-drachm doses of laudanum; headache very great; bowels rather constipated. Croton-oil, one minim; castor-oil, half an ounce, directly; foment the abdomen: tincture of Indian hemp twelve minims; almond mixture one ounce; three times a day. 23rd.-The hemp seems to give him some temporary ease, but still the spasms, or rather convulsions continue, occurring about twice in the twenty-four hours; perfect paralysis on the left side. A blister is ordered to the head, when shaved.

14th.-The blister is risen well; and he seems better, but still relief is not so marked as on the last application of the blister; spasms still continue slightly, paralysis remains. Iodide of potassium, five grains; liquor potassæ, half a drachm; compound decoction of sarsaparilla, an ounce and a half, three times a-day.

June 1st.-B- seems to be entirely relieved from all his spasms, as well as headache; use of his limbs restored; looks quite cheerful, and feels better than he has done for a long time before coming to the hospital. He returns to his meat and wine; continues his medicine, and after remaining in the hospital a month, is discharged at the end of May, apparently in perfect health, still continuing the medicine.

August 6th.-B- saw me at the hospital, and appeared in good health.

11. The last case from which $I$ have given an extract, contrasts with the previous one, inasmuch as its history would seem to point to intestinal irritation as the exciting cause of the disease, and it is only by the treatment and its result, that we can trace the irritation to the brain; for convulsion, and even paralysis, have been consequent upon intestinal worms, and removed together with the exciting cause; and I must confess that was the view that $I$ at first took of the case; but it is to be observed that relief only followed such remedies as were applied directly to the head, under the supposition that the dura mater was in a morbid state, and that relief was then both immediate and complete; but it is only in connexion with other analagous cases that I would venture to take this as evidence. The guard's case also presented both paralysis and convulsion, nor was there any regular epileptic attack in either; but in the guard's all the symptoms clearly belonged to the brain, and the previous history indicated the nature of the attack. In case No 1. there was paralysis with epilepsy, the nature of which could only be conjectured by the result, for it did not differ apparently from those cases which in lunatic asylums have always been considered nearly hopeless, as depending upon organic changes of cerebral structure.

In the absence of post-mortem demonstration, I think $I$ have sufficient circumstantial evidence for attributing such cases as I have described to disease of the dura mater, and for considering that disease as essentially the same as the periosteal disease giving rise to nodes, but $\mathbf{I}$ hope that some additional light may be thrown upon this subject by the resident officers of county lunatic asylums, who must have large opportunity of examining such cases after death.

12. I find these cerebral lesions recognized in their graver forms as syphilitic and the test of their origin asserted to be their yielding to iodide of potassium and sarsaparilla, (see Dr. Todd's Lecture, Medical Gazette). So far as the iodide of potassium test identifies them with periosteal disease, which in these cases affects the dura mater, I believe the distinguished professor to be right, but venture to deny the necessary dependence of periosteal disease on syphilis.

13. My attention has been directed to this subject for a long time, and seven years' practice at the Dreadnought, and seven years at St. Thomas's Hospital, have given me ample opportunity of following it out under peculiar advantages. All my facts have been carefully collected by myself, and noted at the time of examining the patients. It is obvious that a large mass of such details would never be read if published, useless to any one other than the collector, and if they have value, it is by their yielding general propositions, the truth of which depends both upon the accuracy of the observations, and the correctness of the induction. Fortunately, the truth of the propositions may be tested by the correspondence of new facts, not perhaps by every single fact, bearing in mind "that neither one swallow nor one day make a spring;" but we may expect such accuracy as is consistent with the subject matter. All who are conversant with clinical medicine must be aware of the existence of anomalies. With the advancement of knowledge, these anomalies will gradually arrange themselvesunder influences of newly ascertained laws, but in the mean time we must be content with probable, where we cannot obtain absolute, knowledge from induction, provided that the degree of probability is sufficient for our work; but every anomaly lessens the degree of probability of any inductive law, and many such would destroy its probability altogether; though when theyare te in proportion to the normal 
facts, we may still admit the general law, under the supposition that we may have wrongly observed, or wrongly interpreted, the anomalies.

The results of induction from cases of periosteal disease, will form the subject of the next communication.

$$
\text { (To be continued.) }
$$

\section{CASE OF DISEASE OF THE PANCREAS AND LIVER; ACCOMPANIED WITH FATTY DIS- CHARGE FROM THE BOWELS.}

BX ALFRED CLARK, Esq., M.R.C.S.E., L.A.C., Twickenham.

Harriet M-, aged fifty-seven, unmarried, the subject of the following case, had been a sufferer for some years before her last fatal attack to constant derangements of the functions of the liver, and from time to time to attaciss of gall-stones, many of which, no doubt, were passed, but never detected in the fæcal discharges.

About the middle of September, 1850, when away from home, she first began to notice in the urine (and at that time she supposed she passed it with her urine) masses of yellow, greasy-looking matter, in colour, consistence, and general appearance, exactly similar to yellow salt-butter, floating as small solid cakes (about the size of half-crowns) on the surface of the urine as it became cold. On her return home, which was very shortly afterwards, she came again under the care of Mr. Clark, senior, of Twickenham, and I had an opportunity of watching the case throughout. Her general health at the time was much deranged; the bowels very costive; liver inactive; suffering from frequent attacks of acute pain or spasms in the region of the liver, which we referred to her old complaint (gall-stones), and which were relieved by the use of emetics, anodynes, hot fomentations, blue pill, and mild aperients.

About the beginning of October she first mentioned to us the presence of this fatty matter in the urine, and she was herself quite confident that it came from the bladder; but on making careful inquiries, we found that it was present not only in her water, but that all her motions contained more or less of it, and its presence in the urine was dependent on its passing away unconsciously from the rectum whenever she had a call to pass urine, although no fæcal mutter passed at those times.

I gave a specimen of this fatty matterat this time to Dr. Arthur Hassall, who carefully examined it under the microscope, and found it to be perfectly structureless, and in fact could not tell it from a piece of butter. The quantity now voided (October 2nd) was about three or four ounces daily, as nearly as we could guess; the motions were costive, pale-coloured; (but no discoloration of the skin from absorbed bile: there was a deficiency in its secretion;) the motions very offensive; urine scanty and high-coloured; pulse 76 , weak; she herself much emaciated, having been more or less ill for a year previonsly.

After a short time the matter oozed away continuously from the bowels, saturating her linen and bed-clothes; it was of a most offensive odour, and quantities of it were passed with each motion, which had now become very fluid, pale-coloured, and offensive, the fatty matter floating to the surface, and forming a solid cake on cooling; the greatest quantity discharged at any time per diem must have amounted to eight or nine ounces.

During the first month of her illness, she took two or three times a week small doses of blue pill, or mercury-with-chalk, with mild aperients, and the bitter tonics; afterwards the iodide of potassium; but nothing appeared in any way to arrest the discharge of fat, nor did changes in her diet appear to have any effect upon it. Emaciation still continued, but she suffered no pain; in fact, she said she was freer from it than she had been for years.

Nov. 20th.-Dr. Chowne saw her in consultation. His opinion confirmed our own, that there was no doubt disease of the pancreas, whether of a malignant character or not was doubtful, together with a very inactive state of the liver, in all probability dependent on organic changes in its structure; and, more as a palliative than with any hopes of producing a cure, he recommended olive oil in two-drachm doses twice a day, with three grains of mercury-and-chalk every night. We fancied that the oil at first in some measure arrested the discharge, and we increased the dose to six drachms twice a-day, but in a few days it returned with all its former violence.
Dec. 5th.-Still in the same condition; no improvement whatever. In hopes of producing some assimilation of the food taken, (for her appetite was most inordinate,) we gave eight grains of ox-gall, twice or thrice a day, which had the effect of colouring her motions, but with no other visible benefit; she became gradually weaker and weaker, her mind giving way a few days before her death, which took place on the 20 th of January, 1851.

On the 21st of January, eight hours after death, I made a post-mortem examination, assisted by Mr. Clark, Sen., of Twickenham, and Dr. Kershaw, of Kingston. The body was much emaciated; the legs anasarcous. On opening the chest, we found a large quantity of fluid, of a pale-red colour, in the cavities of both pleura, the pleura speckled over with minute spots of tuberculous-looking matter, in a state of softening; the lungs themselves perfectly healthy; no adhesions. Heart soft and flabby; pale-coloured; there was, in fact, fatty degeneration of it; and the pericardium contained about four ounces of fluid. On opening the cavity of the abdomen, we were surprised with the apparently perfectly healthy state of all the viscera; but on removing the omentum from the lower edge of the stomach, and drawing down the intestines, we brought into view a small tumour, about the size of a hen's egg, embedded in some fatty tissue, and situated between the left end of the stomach and the upper end of the spleen, and attached to the extremity of the pancreas. On cutting into this, we found it to be an encysted tumour, containing a mass of dark chocolate-brown substance, yellow in the centre, and glistening with small particles of cholesterine. The pancreas was completely altered in structure, being converted into a mass of nothing else but fatty tissue, with no traces of the original glandular structure. The whole of the liver, pancreas, duodenum, stomach, spleen, and tumour, were now removed from the body, and examined more in detail. The liver was pale, easily broke under the fingers, and presented the usual appearances of a nutmeg liver; gall-bladder very small; the coats much thinned in the posterior part; quite empty of bile, but contained two gallstones of the size of horse-beans; bile-ducts empty; and the ducts in the substance of the liver contained but little bile; the whole liver being rather smaller than natural. The duodenum (perfectly healthy) was now slit open, and the opening of the ductus communis choledochus observed, and a probe being introduced, readily passed into the cavity of the gall-bladder; no opening for the pancreatic duct could be observed; the ductus communis choledochus, and its continuation the cystic duct, were now laid open, and beyond the opening into the duodenum were sufficiently large to admit the little finger; the pancreatic duct did not open into this canal, but where it should have opened into the duodenum, but external to it, we found the remains of the duct, containing in its canal, and completely embraced by it, a mass of calcareous matter, irregular in form, rough, and of a glistening white colour. On carefully cutting down on this, and examining the interior of the duct, we found that it terminated, just external to the duodenum, in a complete cul.de-sac, the canal being pervious towards the pancreas; there was no fluid in the duct; the mass of calcareous matter, consisting in all probability of carbonate and phosphate of lime, had most likely been formed in the pancreas, carried by its auct to its extremity, had failed in passing into the duodenum, and, increasing in size, at last had completely obliterated the duct: during which time, the pancreas, failing in getting rid of its secretion, had been gradually undergoing a change in its structure, and at last was converted into the fatty mass which it presented to us at the post-mortem examination. Whether the liver had acted at all during the last months of her life, is doubtful. The gall-bladder was hardly in the slightest degree stained with bile, nor were any of the ducts. The intestimes generally quite healthy; the kidneys and the uterus healthy; the right ovary diseased, enlarged, and connected with it a small cyst, about the size of an orange, containing a straw-coloured fluid.

Remarks. - The foregoing case is interesting; I think, as. illustrating, in a very marked manner, the connexion of disease of the pancreas with fatty discharge from the bowels; but in this case, as in all others reported, there was an evident deficiency of bile; but the generally healthy state of the other viscera prove that the presence of this fatty matter is depen* dent on absence of the pancreatic fluid, together with deficient supply of the biliary secretion; though in what way this deficiency acts in determining the secretion or couversion of other matters into this fatty substance, it is impossible to say.

Dr. Bright, in the eighteenth volume of the Medico. Chirux 